\title{
Baicalin Promotes Chondrocyte Viability and the Synthesis of Extracellular Matrix through TGF- $\beta /$ Smad3 Pathway in Mouse Chondrocytes
}

Pengzhen Wang

Jinan University

Shaoheng Zhang

Jinan University

Chaosheng Yu

Jinan University

Xifeng Xiong

Jinan University

Aiguo Li

Jinan University

Zhihe Liu ( $\boldsymbol{Z}$ Zliu0731@163.com )

Jinan University https://orcid.org/0000-0002-6697-3732

Research article

Keywords: Baicalin (BA), Smad3, chondrocyte, extracellular matrix

Posted Date: April 27th, 2021

DOI: https://doi.org/10.21203/rs.3.rs-422336/v1

License: () (1) This work is licensed under a Creative Commons Attribution 4.0 International License.

Read Full License 


\section{Abstract}

Background: Osteoarthritis (OA) is epidemic in the elderly people as a common chronic joint disease. By now, drug and surgical treatments are two main therapies for OA worldwidely. Baicalin (BA) is a flavonoid monomer extracted from Scutellaria baicalensis Georgi and is reported that BA has anti-inflammatory, anti-deformation and anti-bacterial effects.

Methods: Micromass culture, alcian blue and Safran $\mathrm{O}(\mathrm{SO})$ /fast green staining were used to investigate chondrocyte viability and ECM synthesis in chondrocytes of all groups. The expression of SOX9 and Smad3 in chondrocytes of all groups were detected by western blot and RT-qPCR, the expression of aggrecan (AGG), type II collagen (Col2a), MMP9/13 and ADAMTS5 were detected by RT-qPCR. In current study, we demonstrated that BA neutralized the down-regulation of chondrocyte viability and extracellular matrix (ECM) secretion, including AGG and Col2a, induced by IL-1 $\beta$. As the key regulators of ECM, the down-regulation of SOX9, and the up-regulation of MMP9/13 and ADAMTS5 induced by IL-1 $\beta$ were abolished by BA. Moreover, BA increased the nuclear translocation and phosphorylation of Smad3, one key mediator of TGF- $\beta$ /Smads pathway. Interestingly, the addition of Smad3 inhibitor SIS3 reversed the promotions of BA on chondrocyte viability, ECM secretion, SOX9 expression, Smad3 nuclear translocation and Smad3 phosphorylation, and the down-regulation of BA on the expressions of MMP9/13 and ADAMTS5.

Conclusions: These results imply that BA can protect chondrocytes against IL- $1 \beta$-induced inflammatory injury through the acceleration of Smad3 phosphorylation and nuclear translocation in chondrocytes. This study demonstrates that BA may be a potential drug for OA clinical treatment.

\section{Background}

Osteoarthritis (OA) is epidemic in the elderly people as a common chronic joint disease [1]. OA has been characterized as joint pain, limb stiffness and others, as well as difficulty in movement which seriously affects the daily travel of patients [2]. The rapid growth of the epidemic brings a great burden on individuals and society [3]. By now, the treatments of OA in the world mainly include drug treatment and surgical treatment [4]. The surgical treatment with high cost is usually used in the advanced stage of OA patients [5], while the drug treatment of OA is trapped in the inhibition the inflammation. The drugs have an advantage in relieve inflammation and pain symptoms, but cannot prevent or delay the progress of OA completely [6]. So, it is urgent and necessary to seek new drugs for OA treatments.

Baicalin (BA) is a flavonoid monomer extracted from Scutellaria baicalensis Georgi. BA has antiinflammatory, anti-bacterial and diuretic effects [7]. It has been reported that BA has a significantly inhibition on the inflammatory response and the expression of IL-1 $\beta$ in chondrocytes [8]. IL-1 $\beta$ is a proinflammatory factor and has shown to inhibit SOX9 expression and extracellular matrix (ECM) synthesis and to induce the productions of MMPs and ADAMTS5 [9]. BA can increase the transcription activity of SOX9, the synthesis of aggrecan (AGG) and type II collagen (Col2a), two main components of cartilage 
ECM [10] and inhibit the expressions of MMPs [11]. SOX9 is a key transcription factor in the development and differentiation of chondrocytes. The decreased expression of SOX9 leads to arrest differentiation of prechondrocytes and serious skeletal deformities [12]. MMPs are proteases and can degrade the ECM [13]. SOX9 is involved in several pathways including TGF- $\beta$ /Smads pathway [14]. TGF- $\beta$ /Smads pathway plays a key role in regulating SOX9 expression during cartilage formation [15]. Recent studies have shown that TGF- $\beta$ /Smads could activate SOX9 expression [16]. As a key component of TGF- $\beta / S$ mads pathway, Smad3 was confirmed to involve in chondrocyte differentiation during cartilage development [17]. TGF- $\beta$ /Smads signaling pathway that results in accelerated chondrocyte maturation is inactivated by de phosphorylation of Smad3 [18]. Since BA can protect chondrocytes by up-regulating the expression of SOX9, but it remains unclear that whether BA protects chondrocytes through the activation of TGF$\beta /$ Samd3 by phosphorylating Smad3. Therefore, a chondrocyte inflammatory model in vitro is established by IL-1 $\beta$ in this study. Cellular and molecular biology methods are used to investigate whether BA protects chondrocyte viability and ECM expression from inflammation through TGF- $\beta / S$ mad3 pathway in mouse chondrocytes.

\section{Materials And Methods}

\section{Reagents}

BA has a chemical formula C33H40015 (Fig. 1a) and it was purchased from Shanghai PureOne Biotechnology Co., Ltd (\#: 21967-41-9, Shanghai, China). BA was dissolved in dimethyl sulfoxide (DMSO) (Sigma Co, St Louis, Mo) and the solution $\left(10^{-2} \mathrm{~mol} / \mathrm{L}\right)$ was stored at $-20^{\circ} \mathrm{C}$. The final concentration of DMSO in the culture was $0.01 \%(v / v)$. IL-1 $\beta$ was purchased from R \& D company (\#: 201-LB-005, NASDAQ, USA). Smad3 inhibitor SIS3 was obtained from MedChemExpress Biological Technology Co. , Ltd (\#: CS-7115. Shanghai, China).

\section{Cell vitality assay}

Mouse chondrocyte (ADTC5) was a kind gift of Dr Yang from Basic Medical College of Jinan University. All cells were grown in DMEM medium (Gibco) containing 10\% (v/v) FBS (Gibco), $100 \mathrm{U} / \mathrm{mL}$ penicillin and $100 \mu \mathrm{g} / \mathrm{mLstreptomycin} \mathrm{(Gibco)}$ in a $5 \% \mathrm{CO}_{2}$ incubator at $37^{\circ} \mathrm{C}$. The chondrocytes were passaged, resuspended and seeded in 96-well plates at a density of $4 \times 10^{3}$ cells/well. Two hundred microliters of DMEM medium with different concentrations of $B A(0,10,20$ and $40 \mu \mathrm{mol} / \mathrm{L})$ or IL-1 $\beta(0,1,2,5$ and 10 $\mathrm{ng} / \mathrm{mL}$ ) were added to the plates respectively next day. After culturing for 24 hours, cell viability was checked by MTS method (Promega Corporation, WI). The absorbance (OD value) at a wavelength of 490 $\mathrm{nm}$ was recorded using a microplate luminescence detector (Promega GloMax 96).

\section{Micromass culture and Alcian blue \& Safranin 0 staining}


Twenty microliters of chondrocyte suspension $\left(1 \times 10^{6}\right.$ cells $)$ was added to 12 -well plates and incubated for 3 hours at $37^{\circ} \mathrm{C}$ for cell attachment. One milliliter of fresh DMEM medium containing various concentrations of drugs were added to each well. The experiments were divided into four groups, namely control group, IL-1 $\beta$ group, IL-1 $\beta+$ BA group and IL-1 $\beta+$ BA + SIS3 group. Every three days, medium was changed. After 2 weeks, aggrecan (AGG) was checked by Alcian blue \& Safranin 0 staining. The staining procedure was as below: wells were washed once with PBS (phosphate buffer saline) and then fixed for $20 \mathrm{~min}$ with $1.0 \mathrm{~mL}$ of $4 \%(\mathrm{v} / \mathrm{v})$ paraformaldehyde (PFA). Subsequently, $0.5 \mathrm{ml}$ of $1.0 \%$ (w/v) Alcian blue (Sigma) (v/v) and $0.5 \mathrm{~mL}$ Safranin 0 solutions were added to each well and incubated for $20 \mathrm{~min}$ at room temperature. After 1 wash with $70 \%(\mathrm{v} / \mathrm{v})$ ethanol and three washes with PBS, photomicrographs of the stained cell mass were obtained by a scanner (EPSON, V550, Japan).

\section{Quantitative reverse transcription polymerase chain reaction (qRT-PCR)}

Chondrocytes $\left(2 \times 10^{5} / \mathrm{mL}\right)$ were cultured in 6 -well plates at $37^{\circ} \mathrm{C}$ in an incubator containing $5 \% \mathrm{CO}_{2}$. After 24 hours of culture, total RNA was extracted using TRIzol reagent (Thermo Fisher Scientific). cDNA was synthesized with a PrimeScriptTM Master Mix reagent kit (Takara Bio, Inc. Japan). qRT-PCR was performed with SYBR Premix ExTaq (Takara Bio, Inc.) using the qTOWER version 3.0 PCR system (Jena Industries, Inc.). The mRNA relative expressions of all target genes were analyzed using a formula ( $2^{-}$ $\triangle \triangle^{T}$ ). GAPDH was used as the internal control. The primer sequences of all genes were shown in Table 1.

Table 1

Sequences of primers used for gene amplification.

\begin{tabular}{|c|c|c|}
\hline Genes & Forward & Reverse \\
\hline GAPDH & 5'-ATTGTGCACCGCAAATGCTT-3' & 5'-ACCACAGCACGATTGTCGAT-3' \\
\hline SOX9 & 5'-TCAACGGCTCCAGCAAGAACAAG-3' & 5'-CTCCGCCTCCTCCACGAAGG-3' \\
\hline Col2a & 5'-GGTCCTCCTGGTCCTGGCATC-3' & 5'-CGTGCTGTCTCAAGGTACTGTCTG-3' \\
\hline AGG & 5'-CGTTGCAGACCAGGAGCAAT-3' & 5'-CTCGGTCATGAAAGTGGCGG-3' \\
\hline MMP9 & 5'-AGAGACCACCACCACCACCAC-3' & 5'-TGCCTGCCTCCACTCCTTCC-3' \\
\hline MMP13 & 5'-CTACCATCCTGCGACTCTTGCG-3' & 5'-CCACATCAGGCACTCCACATCTTG-3' \\
\hline ADAMTS5 & 5'-AAGAGGAGGAGGAGGAGGAGGAG-3' & 5'-AATGGTTGTGAGCTGCCGTATGG-3' \\
\hline
\end{tabular}

\section{Immunohistochemistry}


Chondrocytes $\left(1 \times 10^{5} / \mathrm{mL}\right)$ were seeded on the slides placed on 6-well plates. After 24 hours, the chondrocytes were fixed with PFA for $10 \mathrm{~min}$ at room temperature, and then were incubated with the primary antibody diluent of rabbit anti-p-Smad3 (\# BM4033; 1:50; Boster) overnight at $4^{\circ} \mathrm{C}$. The coverslips were covered with fluorescence secondary antibody (green, \#A32723; 1:200; Thermo Fisher) against light for 30 min at room temperature. Mounting solution with DAPI (\#C1005; Beyotime, China) was used to detect cell nuclei of chondrocytes. Fluorescence was detected by an inverted fluorescence microscope (CX31-32RFL; Olympus).

\section{Western blot}

The chondrocytes $\left(2 \times 10^{5} / \mathrm{mL}\right)$ were cultured in 6-well plates. The experimental groups were as follows: control group; IL-1 $\beta(10 \mathrm{ng} / \mathrm{mL})$ group; IL-1 $\beta(10 \mathrm{ng} / \mathrm{mL})+B A(20 \mu \mathrm{mol} / \mathrm{L})$ group; IL-1 $\beta(10 \mathrm{ng} / \mathrm{mL})+B A$ $(20 \mu \mathrm{mol} / \mathrm{L})+\mathrm{SIS} 3$ blocker $(10 \mu \mathrm{mol} / \mathrm{L})$. The extracted protein was loaded, separated and transferred onto a PVDF membrane by semi-dry transfer method. The membranes blocked by $5 \%$ Bovine Serum Albumin (\# 11021029; Thermo Fisher), were soaked overnight at $4^{\circ} \mathrm{C}$ with solutions of primary anti-SOX9 (\#5173; 1:1000; Cell Signal Technology), anti-p-Smad3 (\# BM4033, 1:50, Boster), Smad3 (\# BA4559; 1:50; Boster) and anti-GAPDH (\#51745; 1:1000; Cell Signal Technology). The membrane was incubated with secondary antibody (\# ARG65351;1:3000; Arigo Biolaboratories) for 1 hour at room temperature next day. Images of the stained-protein bands were recorded using an ECL Western Blotting Substrate (\#32106; Thermo Fisher) kit and quantified using Image lab system version 2.0. (Bio-Rad Laboratories, Inc.).

\section{Statistical analysis}

All data $(n=3)$ were expressed as mean \pm standard deviation and were calculated by SPSS 22.0 software. Student's t-test and One-way ANOVA were used for comparison between two groups and among many groups respectively.

\section{Results}

\section{BA promotes the viability of ADTC5 chondrocytes}

In order to confirm the optimal concentration of BA on chondrocytes viability, the chondrocytes were cultured with increasing concentrations of $B A(0,10,20$ and $40 \mu \mathrm{mol} / \mathrm{L})$ for 24 hours. The chondrocytes viability was analyzed by MTS method. Various concentrations of BA $(10-40 \mu \mathrm{mol} / \mathrm{L})$ increased the cell viability $(p<0.05)$ (Fig. 1b). The most significant promoting effect on chondrocyte activity was found at $20 \mu \mathrm{mol} / \mathrm{L}$ of BA. As shown in Fig. 1c, cell viability was significantly decreased in $10 \mathrm{ng} / \mathrm{mL}$ of IL-1 $\beta$ group compared with control group $(p<0.05)$. 
Combing MTS experiment results and literature [19], $20 \mu \mathrm{mol} / \mathrm{L}$ of BA, $10 \mathrm{ng} / \mathrm{mL}$ of IL-1 $\beta$ and $10 \mu \mathrm{mol} / \mathrm{L}$ of SIS3 were used in subsequent experiments. The experimental groups were divided into 4 groups, namely control group (without any drugs), IL-1 $\beta$ group, IL-1 $\beta+$ BA group and IL-1 $\beta+B A+$ SIS3 group. After 24 hours of culture, cell viability was analyzed by MTS method. As shown in Fig. 1d, the downregulation of chondrocyte viability induced by IL-1 $\beta$ was significantly abolished by BA treatment $(p<$ 0.05). The addition of SIS3 significantly inhibited the protective effect of BA on chondrocyte viability.

\section{BA promotes ECM synthesis in ADTC5 chondrocytes}

Alcian blue and Safranin $O$ staining are classic methods to detect ECM key component AGG [20]. As shown in Fig. 2a, IL-1 $\beta$ treatment obviously down-regulated the expression of AGG in chondrocytes compared with control groups. On the contrary, the down-regulation of AGG induced by IL- $\beta$ was obviously inhibited by BA. Moreover, the addition of SIS3 obviously reversed the protective effect of BA on the expression trend of AGG. The staining results indicated that BA promoted the secretion of $A G G$ in chondrocytes and Smad3 inhibitor SIS3 disturbed the promotion of BA on the secretion of AGG.

By Alcian blue and Safranin 0 staining, we confirmed that BA promoted the expression of AGG in chondrocytes. Since AGG and Col2a are two key components of ECM, we checked the expression of AGG and Col2a in chondrocytes by qRT-PCR. Compared with control group, the expression of AGG and Col2a in IL-1 $\beta$ group was significantly down-regulated $(p<0.05$, Fig. $2 b \& 2 c)$. BA abolished the inhibition of IL$1 \beta$ on AGG and Col2 $\alpha$ expressions $(p<0.05)$. The addition of SIS3 reversed the inhibitory effect of BA on IL-1 $\beta$-induced down-regulation of AGG and Col $2 a(p<0.05)$. These results indicated that BA promoted ECM secretion in chondrocytes and Smad3 inhibitor SIS3 disturbed the promotion of BA on ECM secretion.

\section{BA adjusts the expressions of ECM regulating genes in ADTC5 chondrocytes}

As we know, SOX9, MMPs and ADMTS are key ECM regulators. SOX9 promotes ECM synthesis, and MMPs and ADMTS restrain ECM synthesis. By qRT-PCR and/or western blot, we checked their expressions in chondrocytes. Compared with control groups, the protein expression of SOX9 in IL-1 $\beta$ group was significantly down-regulated ( $p<0.05$, Fig. $3 a \& 3 b)$. BA abolished the inhibition of IL- $1 \beta$ on protein expression of SOX9 $(p<0.05)$. The addition of SIS3 reversed the inhibitory effect of BA on IL-1 $\beta$ induced down-regulation in protein expression of SOX9 $(p<0.05)$. The change in mRNA expression of SOX9 was same as SOX9 protein (Fig. 3c). As shown in Fig. 3d-3f, the mRNA expressions of MMP9, MMP13 and ADAMTS5 in IL-1 $\beta$ group were significantly up-regulated compared with control group, $(\mathrm{p}<$ 0.05). However, the inhibition of IL-1 $\beta$ on the expressions of MMP9, MMP13 and ADAMTS5 was obviously abolished by BA treatment $(p<0.05)$. The addition of SIS3 reversed the protective effect of BA on chondrocytes treated by IL-1 $\beta(p<0.05)$. These results suggested that BA increased the expressions of 
ECM synthesizing genes and decreased the expressions of ECM degrading genes and Smad3 inhibitor SIS3 disturbed the adjusting effect of BA on the ECM regulating genes in chondrocytes.

\section{BA activates Smad3 in ADTC5 chondrocytes}

Since Smad3 inhibitor SIS3 disturbed the promoting effect of BA on the secretion of ECM, it is reasonable to believe that BA may increase the activity of Smad3 which is related with Smad3 phosphorylation and nuclear localization and Smad3 activation may be one of the protective mechanisms of BA on chondrocyte viability and ECM synthesis. The effect of BA on the activation of Smad3 treated by IL-1 $\beta$ was detected by immunofluorescence and western blot. As shown in Fig. 4a, Smad3 was expressed both in the cytoplasm and nucleus of chondrocytes in the control group, while Smad3 was mainly expressed in the cytoplasm of chondrocytes in the IL-1 $\beta$ group. Immunofluorescence results showed that the Smad 3 in the nuclear of chondrocytes in IL-1 1 -induced groups was obviously less than that of BA + IL-1 $\beta$ groups. Oppositely, the addition of SIS3 obviously reversed the nuclear translocation trend of Smad3. On the other side, as shown in Fig. $4 \mathrm{~b} \& 4 \mathrm{c}$, the ratio of $\mathrm{p}-\mathrm{Smad} 3 / \mathrm{Smad} 3$ was down-regulated by IL-1 $\beta$ treatment $(p<0.05)$. But BA treatment neutralized the decrease of $p-S m a d 3 / S m a d 3$ induced by IL-1 $\beta$ treatment $(p<$ $0.05)$. Additionally, in the presence of IL-1 $\beta$ and BA simultaneously, the addition of SIS3 obviously inhibited the protective effect of BA on chondrocytes. These results revealed that BA activates Smad3 in chondrocytes.

\section{Discussion}

$\mathrm{OA}$ is epidemic in the elderly people as a common chronic joint disease [1]. By now, drug and surgical treatments are two main therapies for OA worldwidely [4]. Comparing with the high cost of surgical treatment, drug treatment of $O A$ is more acceptable [5]. These drugs have an advantage in relieve inflammation and pain symptoms. It is reported that BA has anti-inflammatory, anti-deformation, antibacterial and diuretic effects $[7,8]$. In current study, we demonstrate that BA increases the viability of chondrocytes and neutralized the inflammation induced by IL-1 $\beta$ through TGF- $\beta /$ Smad3 pathway.

IL-1 $\beta$ is an inflammatory factor and is related to the progress of OA [20]. It has been shown that IL-1 $\beta$ inhibits the secretion of Col2 $\alpha$ and AGG [21]. In addition, IL-1 $\beta$ stimulates the releases of MMP9/13 and ADAMTS5 in chondrocytes which are responsible for the degradation of ECM $[9,22]$. IL-1 $\beta$ has been also shown to inhibit ECM synthesis through decreasing the expression of SOX9 [9]. By being treated with 10 $\mathrm{ng} / \mathrm{mL}$ of IL-1 $\beta$, we successfully established the inflammatory and injury model of chondrocytes. After treated by IL-1 $\beta$, cell viability and the secretion of Col2 $\alpha$ and AGG were reduced; SOX9, one of ECM synthesis promoter was inhibited; on the contrast, MMP9, MMP13 and ADAMTS5, the ECM degradation promoters, were induced. However, BA significantly reversed the inhibition of IL-1 $\beta$ on the secretion of Col2 $a$ and AGG and the expression of SOX9 which mainly regulates the synthesis of Col2 $a$ and AGG [23$25]$, and decreased the induction of IL-1 $\beta$ on MMP9, MMP13 and ADAMTS5. The promoting effect of BA on the expression of SOX9 and ECM components Col2a and AGG is consistent with previous report [26]. 
The transcriptional activations of SOX9 and TGF- $\beta$ are necessary for prechondrocyte differentiation $[27,28]$. It is reported that TGF- $\beta$ regulates the transcription of SOX9 in human chondrocytes via TGF$\beta / \mathrm{Smad} 2 / 3$ signaling pathway [29]. It is suggested that the overexpression of Smad3 can significantly induce the formation of primary cartilage originated from human mesenchymal stem cell [23]. In addition, Smad3 enhances the transcription activity of SOX9 and Col2a expression, while Smad3 silence inhibits the expression of SOX9 [30]. According to the literature [31], Smad2/3 is associated with SOX9 in TGF- $\beta$ dependent manner and forms a transcription complex with SOX9 in the enhancer region of Col2a. The TGF- $\beta$ /Smad3 pathway plays a key role in SOX9-dependent cartilage formation through up-regulating Smad3 phosphorylation [32]. In this study, BA significantly inhibited the down-regulation of Smad3 phosphorylation and Smad3 cytoplasm transfer induced by IL-1ß. However, the effect of BA on Smad3 phosphorylation and nuclear translocation was blocked by Smad3 inhibitor SIS3. The mechanisms underlying the effect of BA on the phosphorylation and nuclear translocation of Smad3 needs further study to clarify.

\section{Conclusions}

Taken together, present study demonstrates that BA may increase cell viability and cartilage ECM synthesis such as Col2a and AGG. BA increases the expression of SOX9 and inhibits the expression of MMP9, MMP13 and ADAMTS5 by increasing Smad3 phosphorylation and nuclear translocation. This study provides a theoretical basis for BA as a clinical candidate drug for OA treatment, the main results are illustrated in Fig. 5.

\section{Abbreviations}

BA: Baicalin; DMEM: Dulbecco's Modified Eagle Medium; ECM: extracellular matrix FBS: Fetal bovine serum; OA: Osteoarthritis; AGG: Aggrecan; Col2a: Type Il collagen; RT-qPCR: Quantitative reverse transcription polymerase chain reaction.

\section{Declarations}

\section{Acknowledgements}

Not applicable.

\section{Authors' contributions}

PW, AL and ZL designed the study. PW, SZ and XX performed the most of experiments, SZ and XX managed cell culture, $\mathrm{PW}$ analyzed the data. PW, AL and ZL wrote the manuscript. AL and $\mathrm{SZ}$ contributed in the experimental design. All authors have read and approved the final manuscript. 


\section{Funding}

This work was supported by the Medical and Health Science and Technology Project of Guangzhou (20191A011018 \& 20192A010009, PW), the Medical Science and Technology Research Foundation of Guangdong (A2021335, PW), the National Natural Science Foundation of China (81770291, SZ; 81902802, XX), the Guangdong Provincial Natural Science Foundation of China (2018B030311011, SZ), Guangzhou Science and Technology Planning Project (202002030081, SZ), the Medical Science and Technology Research Foundation of Guangdong (B2016018 \& A2018063, XX), the research grants of Guangdong Bureau of Traditional Chinese Medicine (20181206, ZL).

\section{Availability of data and materials}

The datasets used and/or analyzed during the current study are available from the corresponding author on reasonable request.

\section{Ethics approval and consent to participate}

The study was approved by the research ethics committee of Guangzhou Red Cross Hospital, Guangzhou, 510220, China, the approval number is 2019-010-01.

\section{Consent for publication}

Not applicable.

\section{Competing interests}

The authors declare that they have no competing interests.

\section{Author details}

1 Guangzhou Institute of Traumatic Surgery, 2 Department of Cardiology, 3 Department of Otorhinolaryngology, 4 Department of Orthopaedics,Guangzhou Red Cross Hospital, Jinan University, Guangzhou, Guangdong 510220, P.R. China

\section{References}

1. Van den Bosch MHJ. Osteoarthritis year in review 2020: biology. Osteoarthritis Cartilage. 2021;29(2):143-50. https://doi.org/10.1016/j.joca.2020.10.006. 
2. Abramoff B, Caldera FE. Osteoarthritis. Pathology, Diagnosis, and Treatment Options. Med Clin North Am 2020;104:293-311. https://doi.org/ 10.1016/j.mcna.2019.10.007.

3. Khlopas H, Khlopas A, Samuel LT, et al. Current Concepts in Osteoarthritis of the Ankle: Review. Surg Technol Int. 2019;35:280-94. https://pubmed.ncbi.nlm.nih.gov/31237341/.

4. Wellsandt E, Golightly Y. Exercise in the management of knee and hip osteoarthritis. Curr Opin Rheumatol. 2018;30:151-9. https://doi.org/ 10.1097/BOR.0000000000000478.

5. Ferreira de Meneses S, Rannou F, Hunter DJ. Osteoarthritis guidelines: Barriers to implementation and solutions. Ann Phys Rehabil Med. 2016;59:170-3. https://doi.org/10.1016/j.rehab.2016.01.007.

6. Lin X, Chai L, Liu B. Synthesis. Biological Evaluation, and Docking Studies of a Novel SulfonamidoBased Gallate as Pro-Chondrogenic Agent for the Treatment of Cartilage[J]. Molecules 2016, 22(1). https://doi.org/10.3390/molecules22010003.

7. Xing D, Gao H, Liu Z, Zhao Y, Gong M. Baicalin Inhibits Inflammatory Responses to Interleukin-1 $\beta$ Stimulation in Human Chondrocytes. J Interferon Cytokine Res. 2017;37:398-405. https://doi.org/10.1089/jir.2017.0030.

8. Chang CP, Huang WT, Cheng BC, Hsu CC, Lin MT. The flavonoid baicalin protects against cerebrovascular dysfunction and brain inflammation in experimental heatstroke[J]. Neuropharmacology. 2007;52:1024-33. https://doi.org/10.1016/j.neuropharm.2006.10.018.

9. Zhang Y, Li R, Zhong Y, Zhang S, Zhou L, Shang S. Fuyuan Decoction Enhances SOX9 and COL2A1 Expression and Smad2/3 Phosphorylation in IL-1 $\beta$-Activated Chondrocytes[J]. Evidence-based Complementary Alternative Medicine. 2015:821947-7. https://doi.org/10.1155/2015/821947.

10. Huang $X, W u H$, Wang $L$, Zheng $L$, Zhao J. Protective effects of baicalin on rabbit articular chondrocytes in vitro[J]. Experimental Therapeutic Medicine. 2017;13:1267-74. https://doi.org/10.3892/etm.2017.4116.

11. Xing D, Gao H, Liu Z, et al. Baicalin Inhibits Inflammatory Responses to Interleukin-1 $\beta$ Stimulation in Human Chondrocytes. J Interferon Cytokine Res. 2017;37:398-405. https://doi.org/10.1089/jir.2017.0030.

12. Amarilio R, Viukov SV, Sharir A, Eshkar-Oren I, Johnson RS, Zelzer E. HIF1-a regulation of Sox9 is necessary to maintain differentiation of hypoxic prechondrogenic cells during early skeletogenesis[J]. Development. 2007;134:3917-28. https://doi.org/10.1242/dev.008441.

13. Robert S, Gicquel T, Victoni T, Valença S, Barreto E, Bailly-Maître B, Boichot E, Lagente V. Involvement of matrix metalloproteinases (MMPs) and inflammasome pathway in molecular mechanisms of fibrosis. Biosci Rep. 2016;36:e00360. https://doi.org/10.1042/BSR20160107.

14. Coricor G, Serra R. TGF- $\beta$ regulates phosphorylation and stabilization of Sox 9 protein in chondrocytes through p38 and Smad dependent mechanisms. Sci Rep. 2016;6:38616. https://doi.org/10.1038/srep38616.

15. Furumatsu T, Ozaki T, Asahara H. Smad3 activates the Sox9-dependent transcription on chromatin[J]. The International Journal of Biochemistry Cell Biology. 2009;41:1198-204. https://doi.org/10.1016/j.biocel.2008.10.032. 
16. Ciuffreda MC, Malpasso G, Musarò P, Turco V, Gnecchi M. Protocols for in vitro Differentiation of Human Mesenchymal Stem Cells into Osteogenic, Chondrogenic and Adipogenic Lineages. Methods Mol Biol. 2016;1416:149-58. https://doi.org/10.1007/978-1-4939-3584-0_8.

17. Wang X, Zhu Y, Tao H, Jin C, Liu Y, Lu X, Hu X, Fan C. Anbarchian A, et al. Smad2 and Smad3 regulate chondrocyte proliferation and differentiation in the growth plate, PLoS Genet. 2016;12:e1006352. https://doi.org/ 10.1016/j.abb.2014.09.009.

18. Wang X, Zhu Y, Tao H, Jin C, Liu Y, Lu X, Hu X, Fan C. Interaction of ERK1/2 and Smad2/3 signaling pathways in TGF- $\beta 1$-induced TIMP-3 expression in rat chondrocytes. Arch Biochem Biophys. 2014;564:229-36. https://doi.org/10.1016/j.abb.2014.09.009.

19. Chen Z, Zhong H, Wei J, Lin S, Zong Z, Gong F, Huang X, Sun J, Li P, Lin H, Wei B, Chu J. Inhibition of Nrf2/HO-1 signaling leads to increased activation of the NLRP3 inflammasome in osteoarthritis. Arthritis Res Ther. 2019;23:300. https://doi.org/10.1186/s13075-019-2085-6.

20. Yang Z, Gao XJ, Zhao X. CDMP1 promotes type II collagen and aggrecan synthesis of nucleus pulposus cell via the mediation of ALK6. Eur Rev Med Pharmacol Sci. 2020;24:10975-83. https://doi.org/10.26355/eurrev_202011_23581.

21. Hu P, Du J, Zhang S. Oral Administration of Strontium Gluconate Effectively Reduces Articular Cartilage Degeneration Through Enhanced Anabolic Activity of Chondrocytes and Chondrogenetic Differentiation of Mesenchymal Stromal Cells. Biol Trace Elem Res. 2020;193:422-33. https://doi.org/ 10.1093/rheumatology/keu254.

22. Xue M, McKelvey K, Shen K, Minhas N, March L, Park SY, Jackson CJ. Endogenous MMP-9 and not MMP-2 promotes rheumatoid synovial fibroblast survival, inflammation and cartilage degradation[J]. Rheumatology. 2014;12:2270-9. https://doi.org/10.1093/rheumatology/keu254.

23. Sekiya I, Tsuji K, Koopman P, Watanabe H, Yamada Y, Shinomiya K, Nifuji A, Noda M. SOX9 Enhances Aggrecan Gene Promoter/Enhancer Activity and Is Up-regulated by Retinoic Acid in a Cartilagederived Cell Line, TC6[J]. J Biol Chem. 2000; 275: 10738-44. https://doi.org/ $10.1074 / j b c .275 .15 .10738$.

24. Bell DM, Leung KK, Wheatley SC, Ng LJ, Zhou S, Ling KW, Sham MH, Koopman P, Tam PP, Cheah KS. SOX9 directly regulates the type-Il collagen gene[J]. Nat Genet. 1997;16:174-8. https://doi.org/10.1038/ng0697-174.

25. Takimoto A, Kokubu C, Watanabe H, Sakuma T, Yamamoto T, Kondoh G, Hiraki Y, Shukunami C. Differential transactivation of the upstream aggrecan enhancer regulated by PAX1/9 depends on SOX9-driven transactivation. Sci Rep. 2019;9:4605. https://doi.org/10.1038/s41598-019-40810-4.

26. Li Z, Cheng J, Liu J. Baicalin Protects Human OA, Chondrocytes Against. IL-1 $\beta$-Induced Apoptosis and ECM Degradation by Activating Autophagy via MiR-766-3p/AIFM1 Axis. Drug Des Devel Ther. 2020;14:2645-55. https://doi.org/10.2147/DDDT.S255823.

27. Heldin $\mathrm{CH}$, Miyazono K. Dijke P.TGF- $\beta$ signalling from cell membrane to nucleus through SMAD proteins[J]. Nature. 1997;390:465-71. https://doi.org/10.1038/37284. 
28. Shinohara N, Maeda S, Yahiro Y, Sakuma D, Matsuyama K, Imamura K, Kawamura I, Setoguchi T, Ishidou Y, Nagano S, Komiya S. TGF- $\beta$ signalling and PEG10 are mutually exclusive and inhibitory in chondrosarcoma cells. Sci Rep. 2017;7:13494. https://doi.org/10.1038/s41598-017-13994-w.

29. Jahr H, Gunes S, Kuhn AR, Nebelung S, Pufe T. Bioreactor-Controlled Physoxia Regulates TGF- $\beta$ Signaling to Alter Extracellular Matrix Synthesis by Human Chondrocytes. Int J Mol Sci. 2019;20:1715. https://doi.org/10.3390/ijms20071715.

30. Furumatsu T, Tsuda M, Taniguchi N, Tajima Y, Asahara H. Smad3 Induces Chondrogenesis through the Activation of SOX9 via CREB-binding Protein/p300 Recruitment[J]. J Biol Chem. 2005;280:834350. https://doi.org/10.1074/jbc.M413913200.

31. Yang H, Yuan C, Wu C, Qian J, Shi Q, Li X, Zhu X, Zou J. The role of TGF- $\beta 1 /$ Smad2/3 pathway in platelet-rich plasma in retarding intervertebral disc degeneration. J Cell Mol Med. 2016;20:1542-9. https://doi.org/10.1111/jcmm.12847.

32. Coricor G, Serra R. TGF- $\beta$ regulates phosphorylation and stabilization of Sox 9 protein in chondrocytes through p38 and Smad dependent mechanisms[J]. Sci Rep. 2016;6:1-16. https://doi.org/10.1038/srep38616.

\section{Figures}


A<smiles>O=C(O)[C@H]1O[C@@H](Oc2cc3oc(-c4ccccc4)cc(=O)c3c(O)c2O)[C@H](O)[C@H](O)[C@@H]1O</smiles>

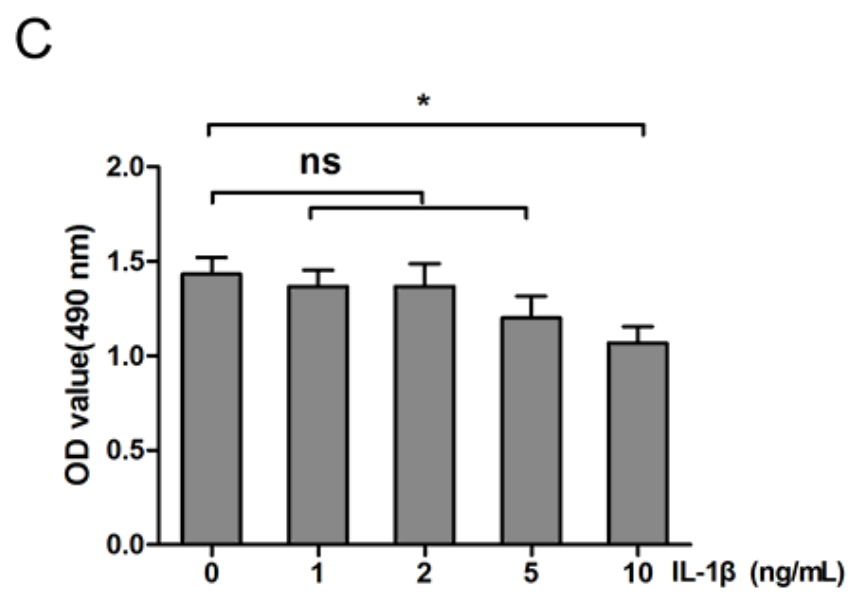

B

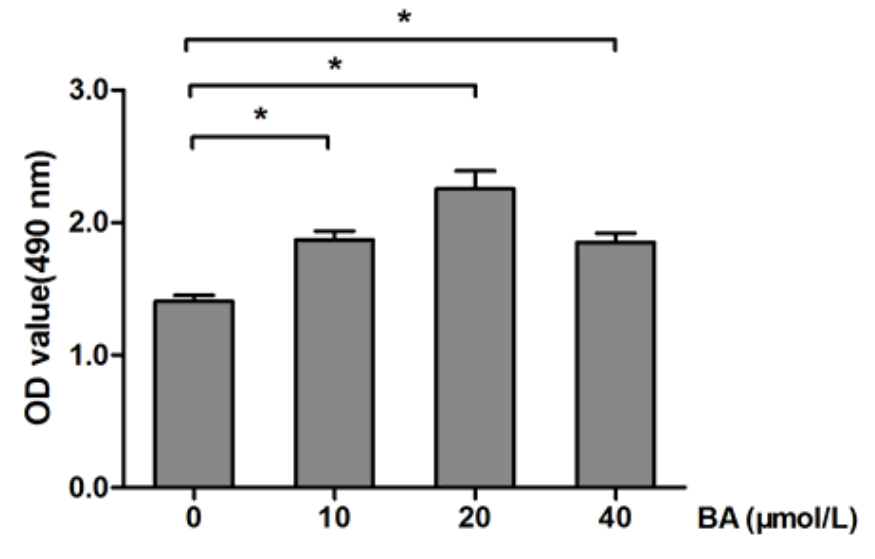

D

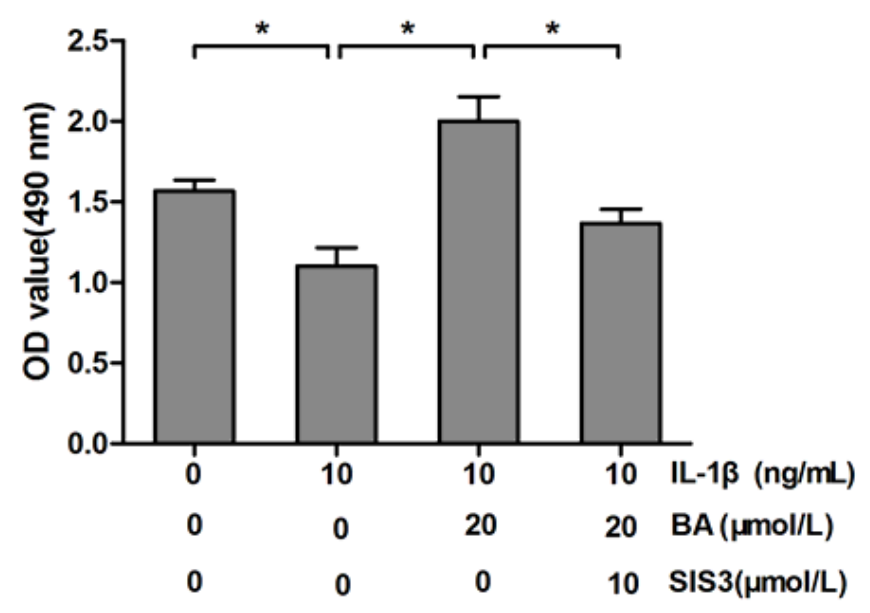

Figure 1

BA promotes the viability of ADTC5 chondrocytes. a Structural formula of BA. $b$ The cell viabilities of ADTC5 chondrocytes which were cultured with increasing concentrations of $B A(0,10,20$ and $40 \mu \mathrm{mol} / \mathrm{L})$ for 24 hours were checked by MTS analysis. $c$ The cell viabilities of ADTC5 chondrocytes which were cultured with increasing concentrations of IL-1 $13(1,2,5$ and $10 \mathrm{ng} / \mathrm{mL})$ for 24 hours were checked by MTS analysis. $d$ The cell viabilities of ADTC5 chondrocytes which were cultured without or with IL-1 $\beta$ (10 $\mathrm{ng} / \mathrm{mL}), \mathrm{BA}(20 \mu \mathrm{mol} / \mathrm{L})$ and SIS3 $(10 \mu \mathrm{mol} / \mathrm{L})$ for 24 hours were checked by MTS analysis

A

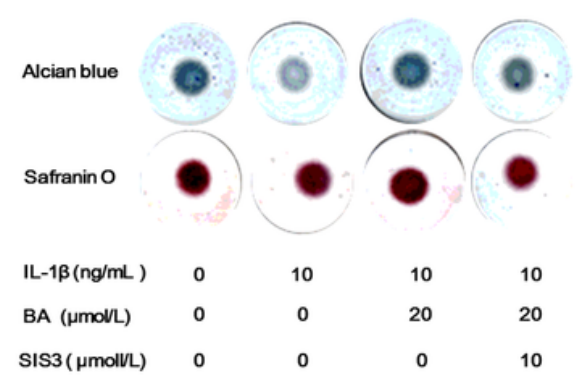

B

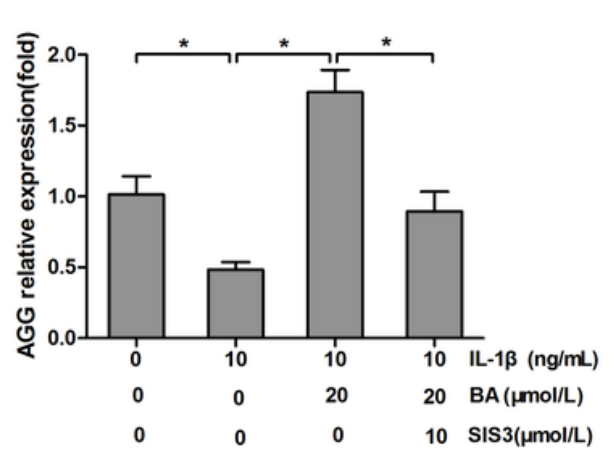

C

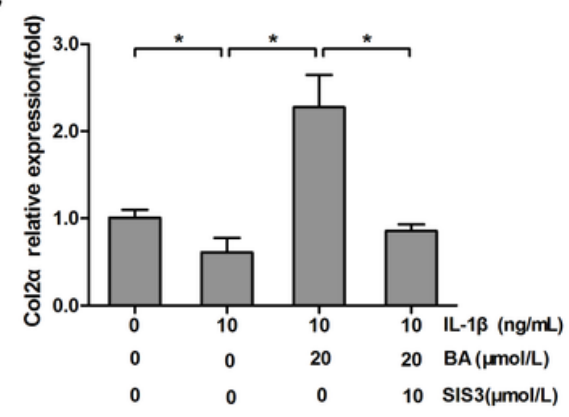


Figure 2

BA promotes ECM synthesis in ADTC5 chondrocytes. a The chondrocyte masses were stained by SO and Alcian blue staining. The relative expression of AGG (b) and Col2a (c) by qRT-PCR in chondrocytes which were cultured without or with IL-1 $\beta(10 \mathrm{ng} / \mathrm{mL}), B A(20 \mu \mathrm{mol} / \mathrm{L})$ and SIS3 $(10 \mu \mathrm{mol} / \mathrm{L})$ for 24 hours. *P < 0.05 compared with corresponding group.
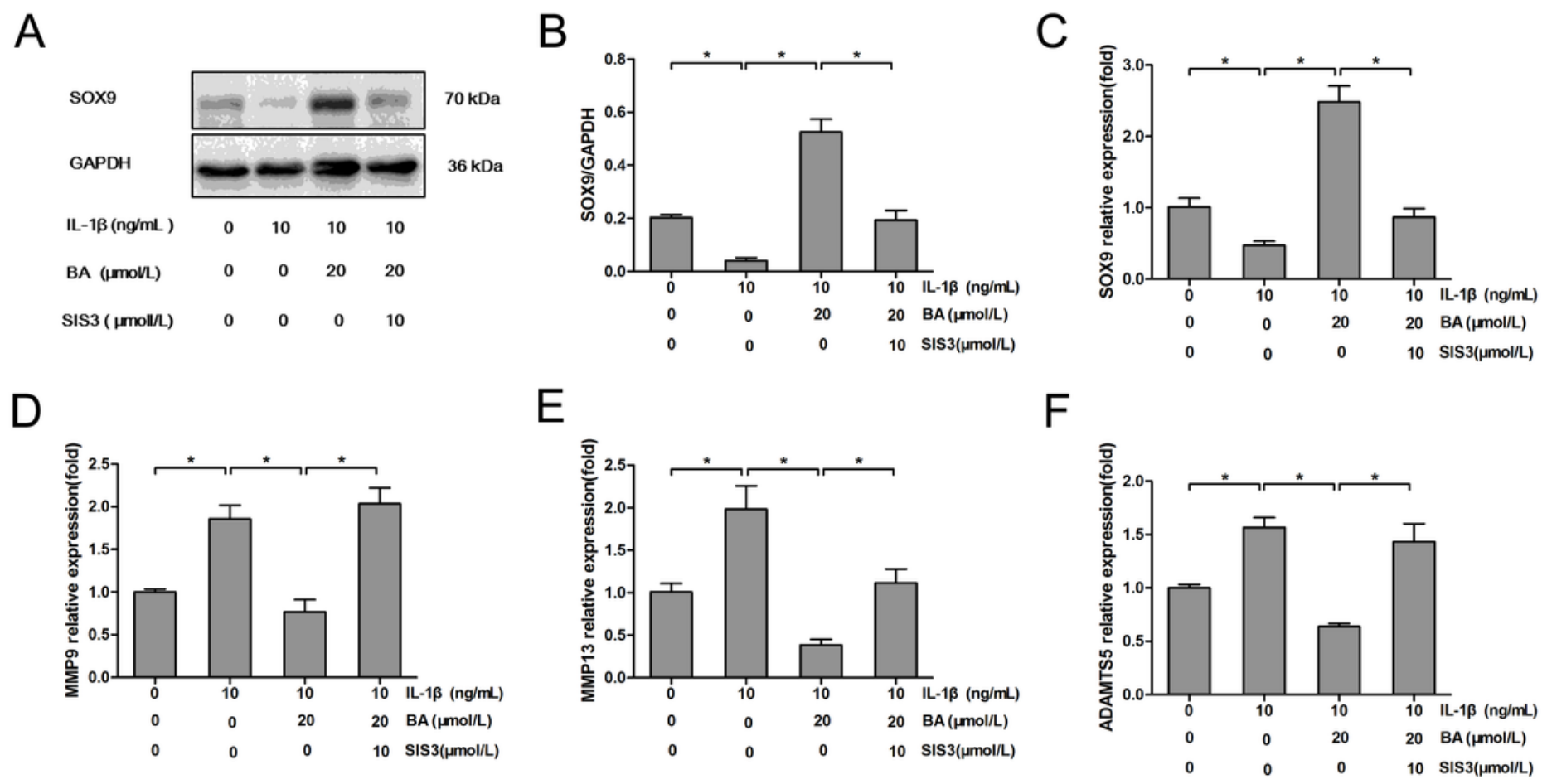

Figure 3

BA adjusts the expressions of ECM regulating genes in ADTC5 chondrocytes. The protein expression of SOX9 (a) and the ratio of SOX9 against GAPDH (b) in chondrocytes which were cultured without or with $\mathrm{IL}-1 \beta(10 \mathrm{ng} / \mathrm{mL}), \mathrm{BA}(20 \mu \mathrm{mol} / \mathrm{L})$ and SIS3 $(10 \mu \mathrm{mol} / \mathrm{L})$ for 24 hours. The relative mRNA expression of SOX9 (c), MMP9 (d), MMP13 (e) and ADMTS5 (f) in chondrocytes which were cultured without or with IL$1 \beta(10 \mathrm{ng} / \mathrm{mL}), B A(20 \mu \mathrm{mol} / \mathrm{L})$ and SIS3 $(10 \mu \mathrm{mol} / \mathrm{L})$ for 24 hours. ${ }^{*} P<0.05$ compared with corresponding group. 
A

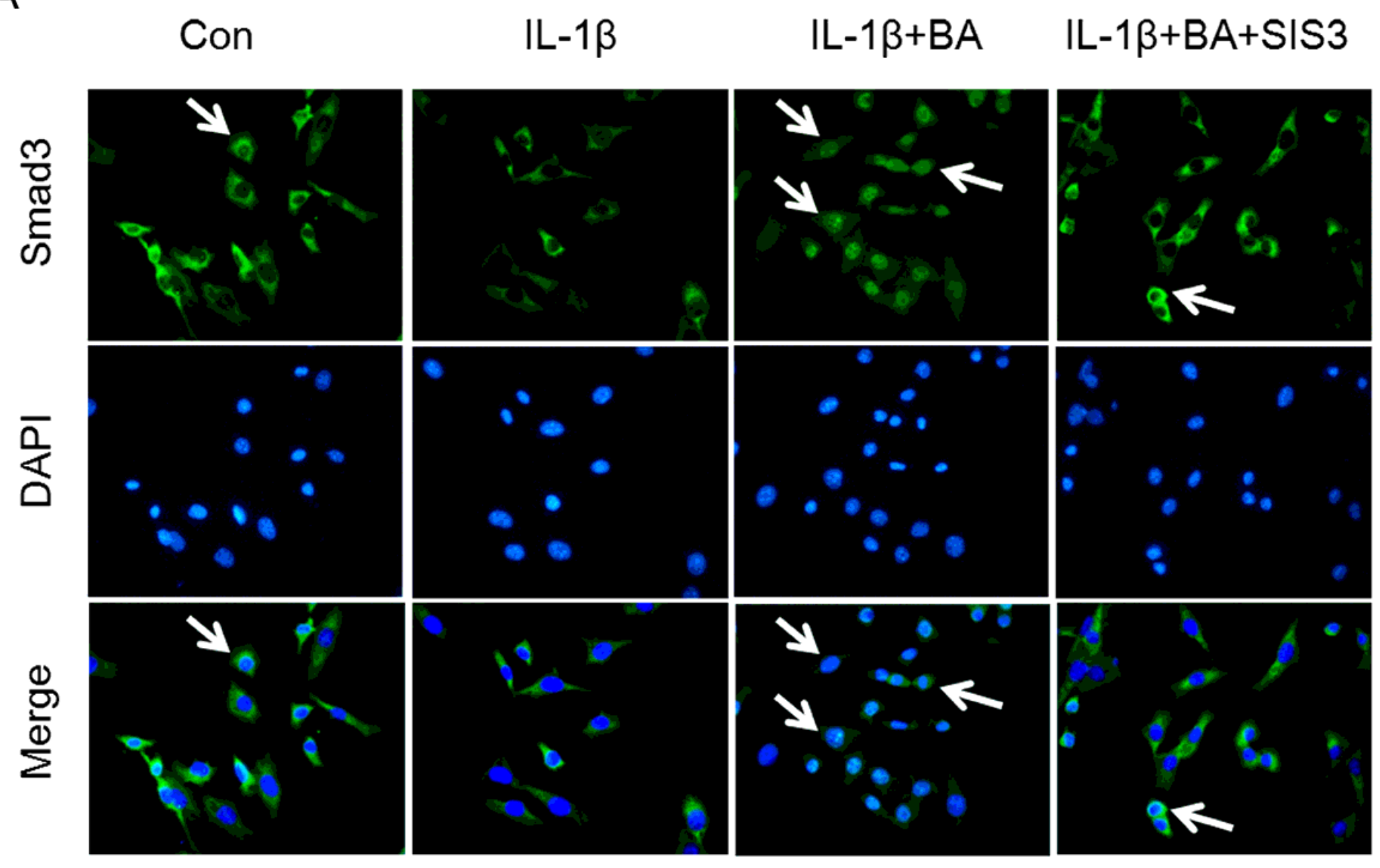

B

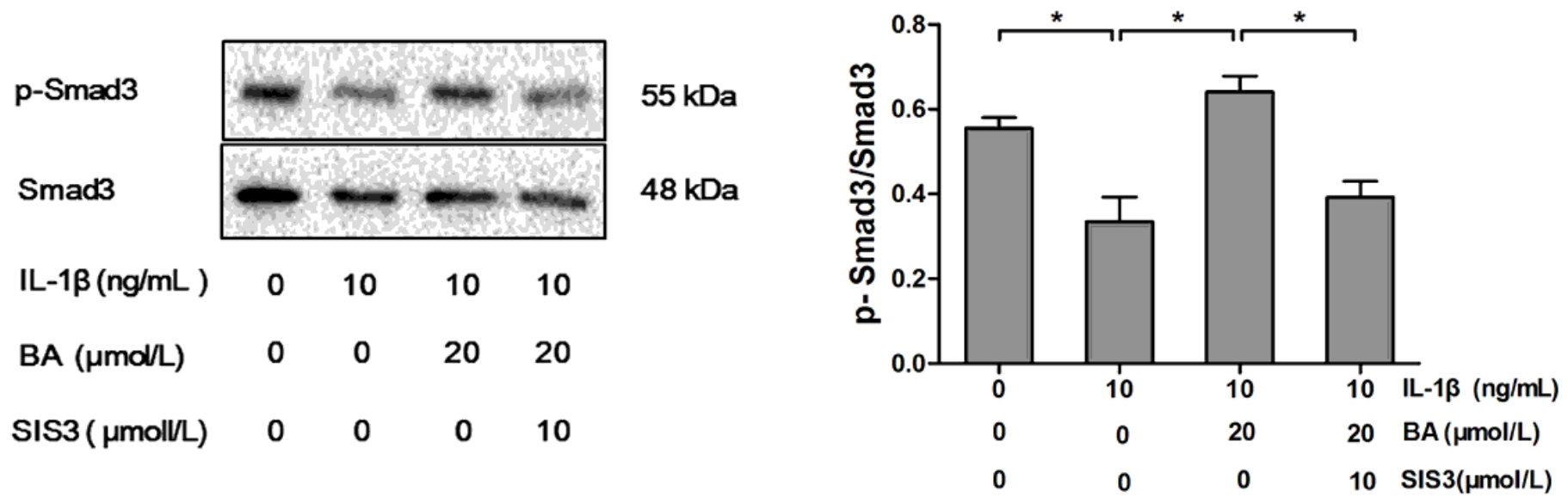

Figure 4

BA activates Smad3 phosphorylation and nuclear localization in ADTC5 chondrocytes. (a) Smad3 localization in chondrocytes which were cultured without or with IL-1 $13(10 \mathrm{ng} / \mathrm{mL}), B A(20 \mu \mathrm{mol} / \mathrm{L})$ and SIS3 $(10 \mu \mathrm{mol} / \mathrm{L})$ for 24 hours checked by immunohistochemistry staining. Smad3 expression (b) and relative phosphorylation (c) checked by western blot in chondrocytes which were cultured without or with $\mathrm{IL}-1 \beta(10 \mathrm{ng} / \mathrm{mL}), \mathrm{BA}(20 \mu \mathrm{mol} / \mathrm{L})$ and SIS3 $(10 \mu \mathrm{mol} / \mathrm{L})$ for 24 hours. ${ }^{*} \mathrm{P}<0.05$ compared with corresponding group. 


\section{Smad3}

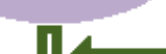

\section{BA}

Cytoplasm
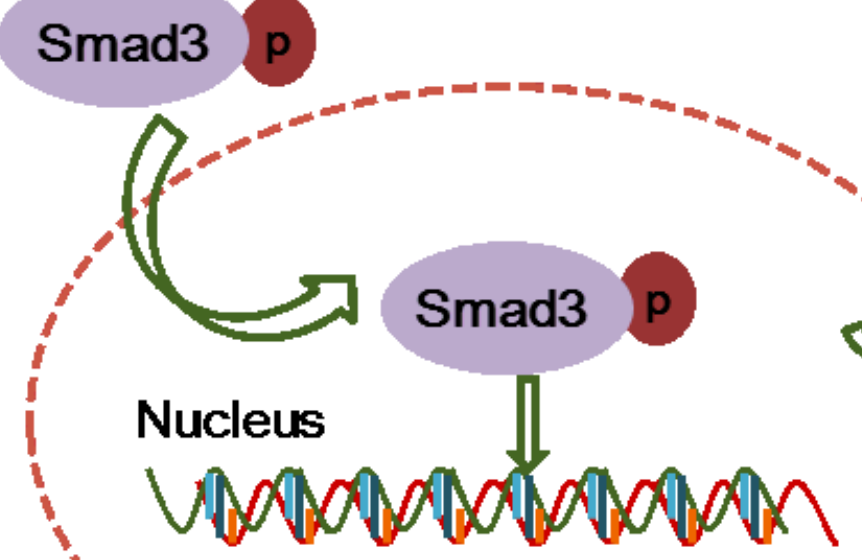

Nucleus

,
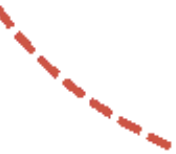

ECM

regulators

介
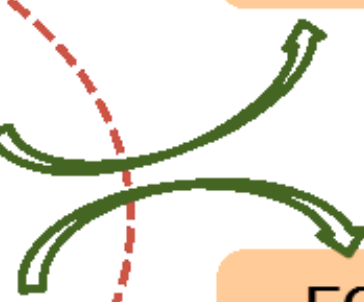

ECM

components

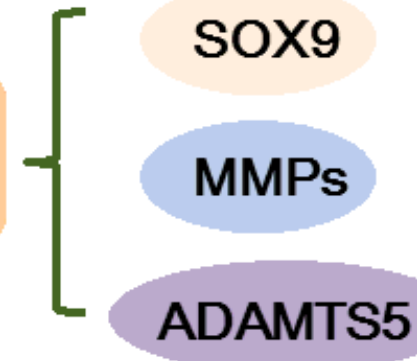

Figure 5

Schematic diagram of research ideas. 